\title{
Optimization of MEMS capacitive accelerometer
}

\author{
Mourad Benmessaoud - Mekkakia Maaza Nasreddine
}

Received: 1 December 2011 / Accepted: 15 January 2013/Published online: 1 March 2013

(C) The Author(s) 2013. This article is published with open access at Springerlink.com

\begin{abstract}
A micro machined accelerometer based on an area variation capacitive sensing for more applications was developed, in this case, we will describe and improve in this work the efficacity as well as the sensitivity of a capacitive accelerometer based on an area of variation capacitive sensing considered as a micro system electro mechanical (MEMS) available and realizable. However, the simulation was performed using MATLAB as software used in complicated situation with an optimization of the several parameters of accelerometer and a single direction, which is consisted with mobile fingers and fixed fingers, as two springs which ensures the damping of the system. The general concept, main design considerations and performance of the resulted accelerometer was optimized and elaborated in order to obtain a good improvement.
\end{abstract}

\section{Introduction}

The micro electro mechanical system (MEMS) technology device design optimization is becoming an interesting and important research issue. However, various efforts on MEMS device design optimization and automation have been made as modeling and simulation of a capacitive micro machined accelerometer.

Compatibility with conventional CMOS provides advantages high yield and fast prototyping that should be adjustable and transferable to any CMOS foundry.

M. Benmessaoud $(\varangle) \cdot$ M. M. Nasreddine Department of Electronic Engineering, Faculty of Electrical Engineering, University of Science and Technology of Oran, BP 1505, EL M'Naouer, Oran 31000, Algeria

e-mail: benmessaoud_mourad@yahoo.fr
In this work, we present the difference and the relationship between the design optimization of a capacitance folded beam MEMS comb accelerometer device and the device sensitivity such as beam width, beam length, mass width. Based on the analysis, an optimized design of the MEMS comb capacitive accelerometer device is suggested.

\section{CMOS micromachining process}

The CMOS (complementary metal oxide semiconductor) micromachining accelerometer uses high technology, are made from custom processes combining polysilicon surface micromachining and electronic circuits processes (Xie and Fedder 2002). It is fabricated using three-metal $0.5 \mu \mathrm{m}$ n-well CMOS process through MOSIS (Luo et al. 2002). After the fabrication, two dry etch steps, shown in Fig. 1, are used to define and release the structure. Figure 1a shows the cross section of the chip after regular CMOS fabrication. In the first step of post processing as shown in Fig. 1b, dielectric layers are removed by an anisotropic $\mathrm{CHF}_{3} / \mathrm{O}_{2}$ reactive-ion etch (RIE) with the top metal layer acting as an etch resistant mask (Zhang 1994).

After the sidewall of the microstructure is precisely defined, an isotropic $\mathrm{SF}_{6} / \mathrm{O}_{2}$ (RIE) is performed to etch away the bulk silicon and release the composite structure as shown in Fig. 1c (Zhang et al. 1999). Layout in the metal layers is designed to form beams, plates, and electrostatic comb fingers. Material property values for the composite structures include a density of $2,300 \mathrm{~kg} / \mathrm{m}^{3}$ and a Young's modulus of $62 \mathrm{GPa}$.

Electrically isolated multi-layer conductors can be routed in the composite structures, enabling more design options (compared to homogeneous conductor structures). For example, electrically decoupled sensing and actuating 


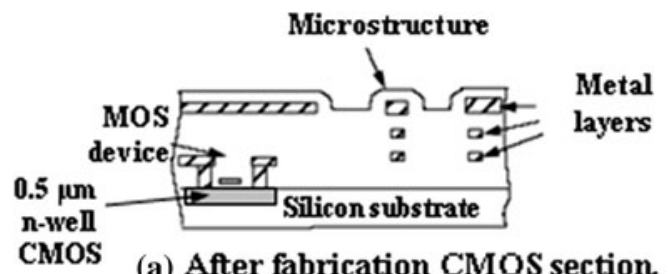

(a) After fabrication CMOS section.

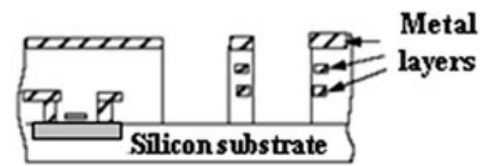

(b) After dielectric etching.

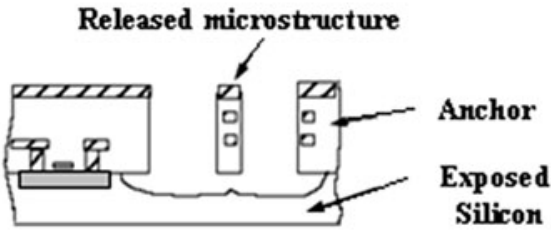

(c) After bulk silicon etching

Fig. 1 CMOS-MEMS micromachining process (Luo et al. 2002; Zhang 1999)

comb fingers may be built on the same structure and fullbridge capacitive differential and common centroid combfinger designs can be readily implemented (Luo et al. 2002).

A full-bridge capacitive sensor has double transducer sensitivity of a half-bridge. Higher transducer sensitivity improves the signal-to-electrical noise ratio. At the same time, since the full-bridge capacitive sensor has differential output, it has better ability to reject common mode noise. The undercut of silicon in the release step (Fig. 2) constrains the placement of sensing circuits to at least $15 \mu \mathrm{m}$ away from the microstructures. Compared to most commercialized polysilicon micromachining technology, the
MEMS to electronics interconnect in CMOS-MEMS is shorter, and suffers less parasitic capacitance. Such parasitic on high impedance wiring can be made small relative to input capacitance of interface circuits, so the transducer sensitivity is larger when capacitive sensing is employed.

$C_{\text {para }}$ is the parasitic capacitance and $C_{1}, C_{2}, C_{3}, C_{4}$ represent the differential capacities between the movable fingers and the sensing fingers.

Figure $2 \mathrm{a}, \mathrm{b}$ represent the schematic of accelerometer and the equivalent model. The topology used here is that of a single axis, common centroid, fully differential, capacitive sensing lateral accelerometer (Zhang 1994). The proof mass is suspended using four serpentine springs attached to its corners. Interdigitated comb drives are used for differential capacitive sensing as shown in Fig. 2a. Each finger consists of two electrical nodes, one each for the two capacitors on the half capacitive bridge; and the sense nodes are located on the stator fingers. This is used to create a common centroid configuration, which is not possible in polysilicon MEMS. In order to counter out of plane curl mismatch between the comb fingers, the fingers are attached to a peripheral frame rather than being anchored to the substrate. The different schemes of capacitive interfaces are represented in the Fig. 3.

The most commonly used capacitive sense interface is a single-ended half-bridge interface shown in Fig. 3a. Change in capacitance can be measured by driving the ends of the bridge and taking the central node as the output. Fully differential interfaces are always preferred to their single-ended counterparts because of better power supply rejection and first-order cancellation of substrate coupling. Usually, differential capacitive sense interfaces have been implemented with polysilicon surface micromachining processes. In some designs displacement is sensed with a capacitive half-bridge by modulating the central node (the proof mass) and connecting the two fixed ends to a differential position sense interface as shown in Fig. 3 b.
Fig. 2 Differential capacitor structure and Equivalent schematic model of accelerometer (Luo et al. 2002; Zhang et al. 1999)
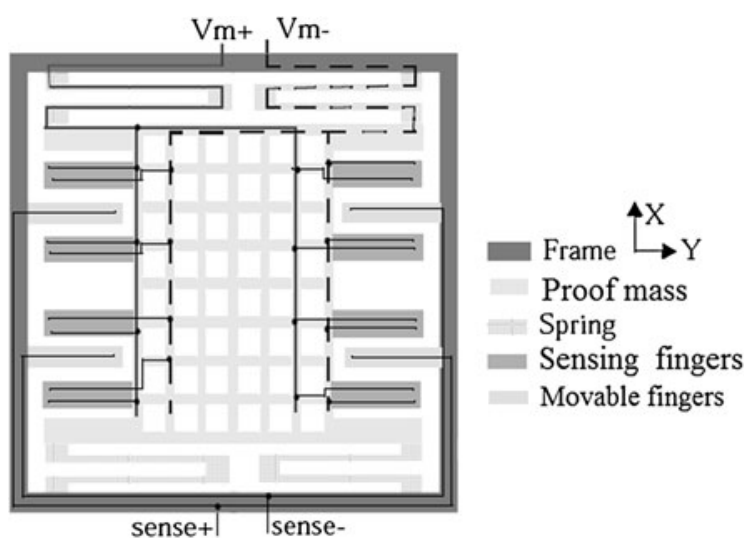

(a)

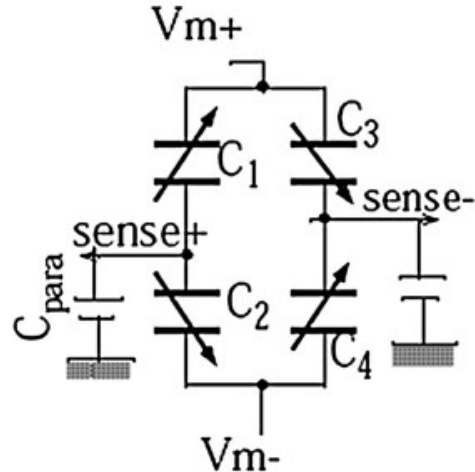

(b) 


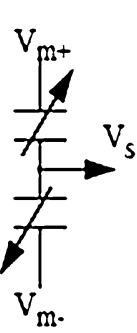

(a)

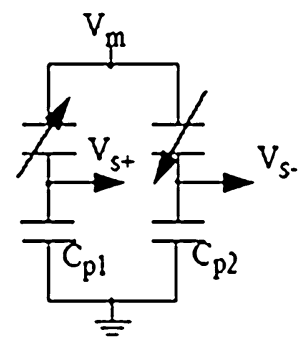

(b)

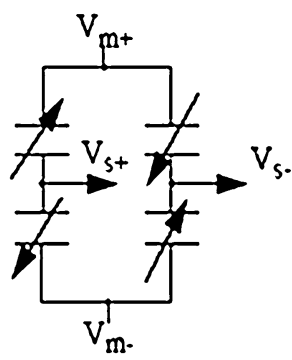

(c)
Fig. 3 Different schemes of capacitive interfaces (Zhang 1994)

Since there is only one modulation node instead of two differential ones, a significant common-mode signal will appear at the input nodes of the differential interface.

The difference between two parasitic capacitors $\left(C_{p 1}, C_{p 2}\right)$ results in output offset which can be a great source of drift over environmental variations, such temperature and aging.

\section{Principle of operation}

A schematic of a capacitive micro accelerometer simplified is shown in Fig. 4. The central part of the accelerometer is a suspended micromechanical proof mass. When an external acceleration is applied, the proof mass will move with respect to the moving frame of reference which acts as the sensing element (Zhang 1994).

The displacements of the proof mass imply an acceleration which can be measured by several methods. For the capacitive sensing approach, the displacement is detected by measuring the capacitance change between the proof mass and adjacent fixed electrodes. Low parasitic capacitance achieved from monolithic integration is the key to maximizing the performance with this technique.

On the basis of the mechanical parameters schematic for the sensing element shown in Fig. 4, the differential equation for the displacement $x$ as a function of external acceleration is that of a second-order mass-spring-damper system (Luo et al. 2002; Zhang 1994):

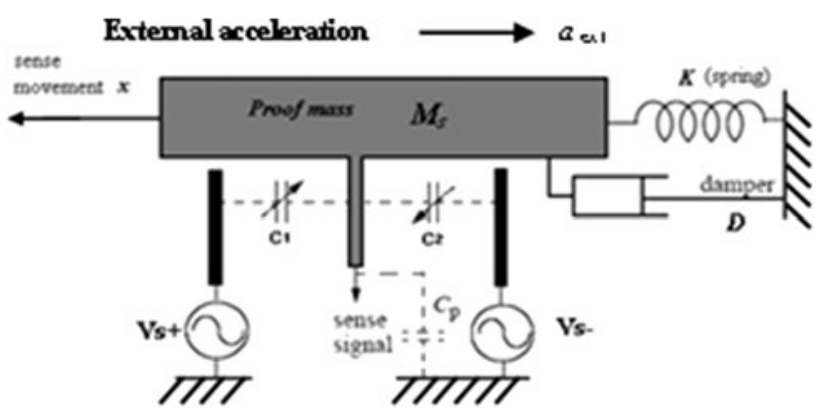

Fig. 4 Schematic of a capacitive micro accelerometer (Zhang 1994)
$M_{s} \cdot \frac{d^{2} x}{d t^{2}}+D \cdot \frac{d x}{d t}+K_{s} \cdot x=M_{s} \cdot a_{e x t}$

where $K_{s}$ is the spring constant, $D$ is the damping coefficient, $M_{s}$ is the proof mass and $a_{e x t}$ is the external acceleration.

With Laplace transform notation, the above equation converts to a second-order transfer function:

$\frac{X(s)}{A(s)}=\frac{1}{s^{2}+s \cdot \frac{D}{M_{s}}+\frac{K_{s}}{M_{s}}}=\frac{1}{s^{2}+s \cdot \frac{\omega_{r}}{Q}+\omega_{r}^{2}}$

where $\omega_{r}$ is the resonant frequency, $Q$ is the quality factor.

At low frequency $(\omega \ll \omega r)$ :

$\frac{X}{A} \approx \frac{1}{\omega_{r}^{2}}$

The sensitivity is inversely proportional to the square of the resonant frequency which means the lower the resonant frequency the higher the sensitivity. But actually, the lower limit of resonant frequency is bounded by many factors such as the mechanical shock resistance, the achievable lowest spring constant, the highest possible effective mass, and manufacturability.

\section{Device design}

The structure design of a poly-silicon surface-micromachined MEMS comb accelerometer is shown in Fig. 5.

The movable parts of this MEMS comb accelerometer consist of four folded-beams, a proof mass and some movable fingers. The fixed parts include two anchors and some left/right fixed fingers. The central movable mass is connected to both anchors through four folded beams.

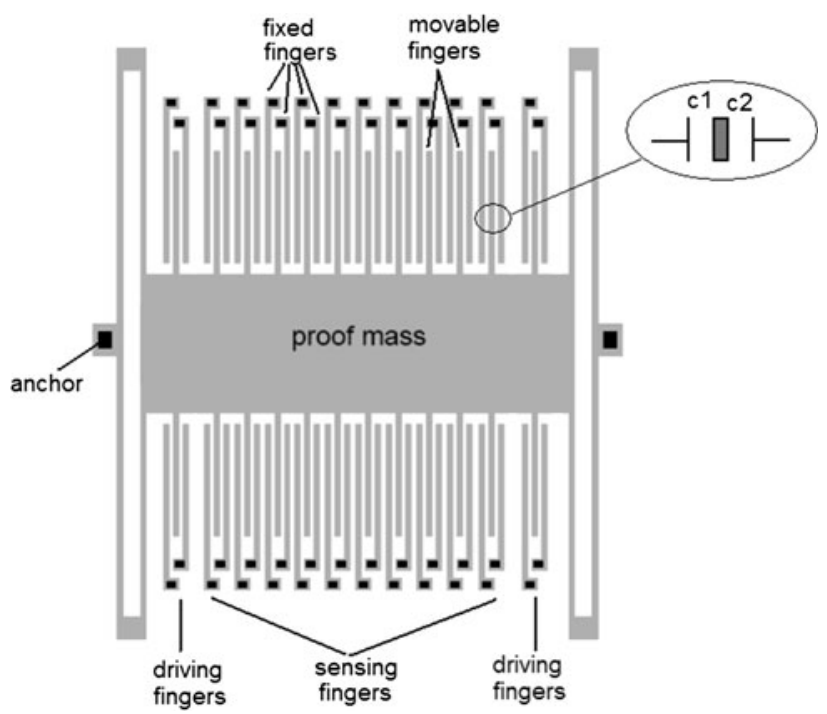

Fig. 5 The general designs of MEMS comb accelerometer (Sharma et al. 2012) 
In the right and left side of the each movable finger, there are left and right fixed fingers. The movable fingers constitute the differential capacitance pair $C_{1}$ and $C_{2}$ with left and right comb fingers (Sharma et al. 2012).

If is no acceleration $(a=0)$, the movable fingers are resting in the middle of the left and right fixed fingers, the left and right capacitance pairs $C_{1}$ and $C_{2}$ are equal. When is any acceleration $a$ along horizontal direction parallel to the device plan, the proof mass $M_{s}$ experiences an inertial force become $-M_{s} \cdot a$ along the opposite direction. However, the beams deflect and the movable mass and movable fingers move for a certain displacement $x$ along the direction of the inertial force. That automatically changes the left and right capacitance gaps; hence the differential capacitances $C_{1}$ and $C_{2}$ will also be changed. One can know the value and direction of acceleration one measuring the difference of the capacities change.

When there is no acceleration, a driving voltage $V_{d}$ is applied to the left or right fixed driving fingers.

The electrostatic force will attract the movable fingers toward the left or right direction. By measuring this displacement and comparing with good device response, one knows whether the device is good or faulty.

\section{Mechanical suspension}

The topology of folded beam with turns can provide a lower spring constant, and thus higher sensitivity.

The spring constant of this structure is:

$K_{s}=\frac{1}{2} \cdot E \cdot h \cdot\left(\frac{w_{b}}{l_{b}}\right)^{3}$

The four folded beam can be treated as four springs connected in parallel. Therefore, the spring constant along the $\Delta x$ direction for a suspension structure as shown in Fig. 6, can be determined as:

$K_{\text {total }}=2 \cdot E \cdot h \cdot\left(\frac{w_{b}}{l_{b}}\right)^{3}$

where $K s$ is the constant of spring for one folded beam, $l_{b}$ is the beam length, $w_{b}$ is the beam width, $h$ is the beam thickness, $E$ is the Young's modulus of the structural material.

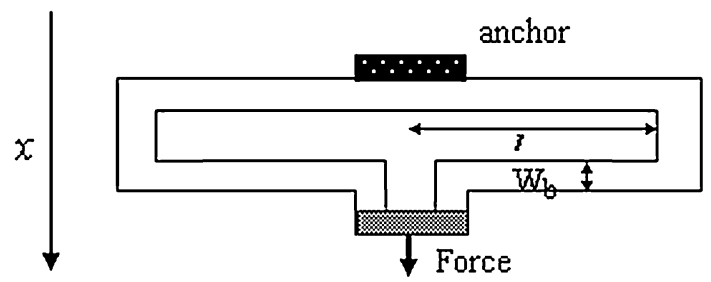

Fig. 6 Detail of accelerometer Spring structure (Kuan 2008)

\section{Damping and quality factor}

There are two categories of damping mechanisms. First, structural damping is caused by friction within composite structural (Zhang 1994). The second is viscous air damping at atmospheric pressure. For the lateral accelerometer, squeeze film damping which occurs when the air gap between two closely placed parallel surfaces changes, is not critical either.

The damping coefficient between a single comb finger gaps is giving by (Amini 2006)

$D=N_{f} \cdot \eta_{\text {eff }} \cdot l_{b} \cdot\left(\frac{h}{d_{0}}\right)^{3}$

$N_{f}$ total sensing finger number, $\eta_{\text {eff }}$ is the effective viscosity of air, $d_{o}$ capacitance gap.

However the quality factor is given by:

$Q=M_{s} \cdot \omega_{r} / D$

where $\omega_{r}=\sqrt{K_{s} / M_{s}}$, reducing the damping increases the possibility of resonant behavior (high Q).

\section{Basic knowledge for capacitive MEMS}

\subsection{Devices}

A typical MEMS differential capacitance structure is shown in Fig. 7, where $M_{s}$ represent the movable plate mass; $F_{1}$ and $F_{2}$ denote fixed or fingers plates, while $B_{1}$ and $B_{2}$ are both beams of the MEMS device (Xiong 2005).

The movable plate $M_{s}$ is anchored to the substrate through two flexible beams $B_{1}$ and $B_{2}$. It constitutes differential capacitances $C_{1}, C_{2}$ with the top and bottom fixed plates. In the static mode, the movable plate $M_{S}$ is located in the center between $F_{1}$ and $F_{2}$, therefore:

$C_{1}=C_{2}=\frac{\varepsilon_{0} \cdot S}{d_{0}}$

where $\varepsilon_{0}$ is the dielectric constant of air, $S$ is the overlap area between $M_{s}$ and $F_{1}, F_{2}, d_{0}$ is the static capacitance gap between $M_{s}$ and $F_{1}, F_{2}$.

When there is acceleration will result in the deflection of beams and a certain displacement of movable plate $M_{s}$

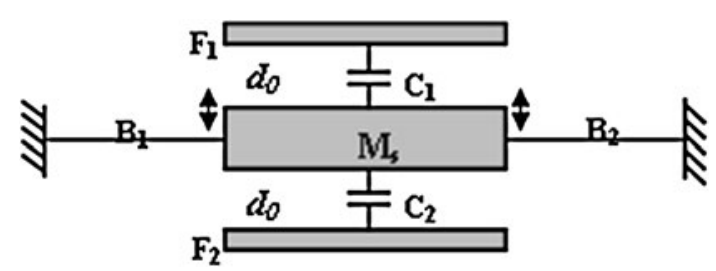

Fig. 7 Schematic diagram of a capacitive MEMS device 
along the vertical direction. Assume the central movable mass moves upward with a displacement of $x$. Given ( $\left.x \ll d_{0}\right), C_{1}$ and $C_{2}$ under the test stimuli can be derived by:

$C_{1}=\frac{\varepsilon_{0} \cdot S}{\left(d_{0}-x\right)} \approx \frac{\varepsilon_{0} \cdot S}{d_{0}}\left(1+\frac{x}{d_{0}}\right)$

$C_{2}=\frac{\varepsilon_{0} \cdot S}{\left(d_{0}+x\right)} \approx \frac{\varepsilon_{0} \cdot S}{d_{0}}\left(1-\frac{x}{d_{0}}\right)$

As there will be a displacement $x$ of the movable plate $M_{s}$, modulation voltage $V_{m p}$ and $V_{m n}$ are applied to $F_{1}$ and $F_{2}$ separately:

$V_{F 1}=V_{m p}=V_{0} \operatorname{sqr}(\omega \cdot t)$

$V_{F 2}=V_{m n}=-V_{0} \operatorname{sqr}(\omega \cdot t)$

where $V_{0}$ the modulation voltage amplitude, $\omega$ the frequency of the modulation voltage, $t$ the time for operation.

According to the charge conservation law, the charge in capacitances $C_{1}$ and $\mathrm{C}_{2}$ must be equal, so we have:

$C_{1}\left(V_{F 1}-V_{M}\right)=C_{2}\left(V_{M}-V_{F 2}\right)$

where $V_{M}$ is the voltage level sensed by the movable plate $M_{s}$. Solving the above equations, we have:

$V_{M}=\left(\frac{x}{d_{0}}\right) \cdot V_{0} \cdot \operatorname{sqr}(\omega \cdot t)$

The central movable plate $M_{s}$ acts as a voltage divider between the top and bottom fixed plates $F_{1}$ and $F_{2}$ respectively. By measuring the voltage level on central movable electrode $V_{M}$, we can find the displacement $x$ of the central movable plate $M_{s}$, which in turn is directly proportional to the physical stimuli. Thus, we can derive the value of the applied physical stimuli. This is the working principle for most differential capacitive $M E M S$ devices. If voltage $V_{d}$ is applied to the fixed plate $F_{1}$ and nominal voltage $V_{\text {nominal }}$ is applied to $M_{s}$, an electrostatic attractive force $F_{d}$ will be experienced by the central movable mass:

$F_{d}=\frac{\varepsilon_{0} \cdot S \cdot V_{d}^{2}}{2 \cdot d_{0}^{2}}$

For vertical electrostatic driving, the driving voltage cannot exceed a threshold value by which the deflection exceeds $1 / 3$ of the capacitance gap $d_{0}$. Otherwise, the movable plate will be stuck to the fixed plate through a positive feedback, and a short circuit will occur.

\section{Analysis of the device}

When an acceleration $a$ along the horizontal direction parallel to the device plan is applied to the accelerometer, the beam deflects under the effect of inertial force. The deflection of beam is in opposite direction of the applied acceleration. The displacement sensitivity of the device is defined as the displacement of the movable mass and movable fingers per unit gravity acceleration $g$ along devices sensitive direction. The beam-mass structure of the accelerometer can be treated as a simplified spring-mass model. The four folded beam can be treated as four springs connected in parallel.

In order to find out the sensitivity of a comb accelerometer, dynamic analysis must be performed. A MEMS comb accelerometer actually can be simplified by a springmass model. For each folded-beam, both sections of the beam can be treated as two springs connected in series. Each beam section can be treated as double-clamped beam model.

Assume for each section of the folded-beam, the beam width and length is $W_{b}$ and $L_{b}$ separately. The width and length of central proof mass are $W_{m}$ and $L_{m}$ separately. The device thickness (thickness of the poly-silicon layer) is $h$. There is $N_{f}$ totally sensing finger groups. For each movable finger, the finger width and length are $W_{f}$ and $L_{f}$ separately. When there is no acceleration, the capacitance gap between each movable finger and its left/right fixed fingers is $d_{0}$. The density $\rho$, Young's modulus $E$ of polysilicon material and unit gravity are given as below (Xiong 2005).

Considering that the length not covered with mobile finger is null $\left(\Delta l_{f}=0\right)$ as shown in Fig. 8.

The static sensing capacitance of the MEMS comb accelerometer when there is no acceleration $(a=0)$ is:

From Eq. (8) we have (Sharma et al.):

$C_{10}=C_{20}=C_{0}=\frac{\varepsilon_{0} \cdot N_{f} \cdot L_{f} \cdot h}{d_{0}}$

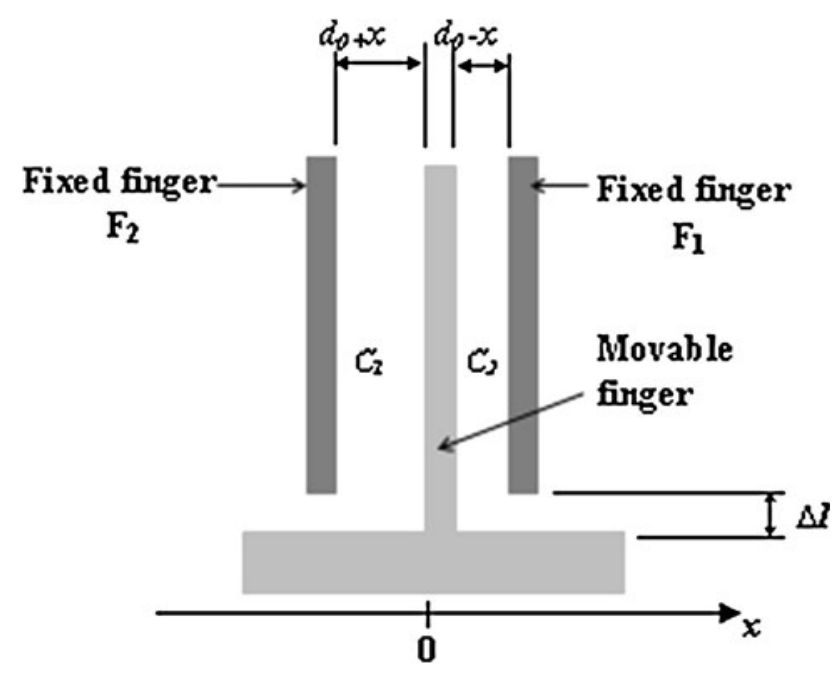

Fig. 8 Differential capacitance of MEMS comb accelerometer (Xiong 2005) 
When there is acceleration $(a \neq 0)$ along left direction horizontally; the movable mass experiences an inertial force toward right by $x$ (Fig. 4). Assume small deflection approximation $\left(x \ll d_{0}\right)$, the left (right) capacitances $C_{1}$, $C_{2}$ are changed to:

$$
\begin{aligned}
C_{1} & =\frac{\varepsilon_{0} \cdot N_{f} \cdot L_{f} \cdot h}{\left(d_{0}+x\right)}=\frac{\varepsilon_{0} \cdot N_{f} \cdot L_{f} \cdot h}{d_{0} \cdot\left(1+x / d_{0}\right)} \\
& \approx \frac{\varepsilon_{0} \cdot N_{f} \cdot L_{f} \cdot h}{d_{0}} \cdot\left(1-\frac{x}{d_{0}}\right) \\
C_{2} & =\frac{\varepsilon_{0} \cdot N_{f} \cdot L_{f} \cdot h}{\left(d_{0}-x\right)}=\frac{\varepsilon_{0} \cdot N_{f} \cdot L_{f} \cdot h}{d_{0} \cdot\left(1-x / d_{0}\right)} \\
& \approx \frac{\varepsilon_{0} \cdot N_{f} \cdot L_{f} \cdot h}{d_{0}} \cdot\left(1+\frac{x}{d_{0}}\right)
\end{aligned}
$$

The differential capacitance change $\Delta C$ is:

$$
\begin{aligned}
\Delta C & =C_{1}-C_{2}=\frac{2 \cdot \varepsilon_{0} \cdot N_{f} \cdot L_{f} \cdot h}{d_{0}} \cdot\left(\frac{x}{d_{0}}\right) \\
& =2 \cdot C_{0} \cdot\left(\frac{x}{d_{0}}\right)
\end{aligned}
$$

From Eqs. (17) and (18), we obtain for small deflection approximation, differential capacitance change $\Delta C$ is directly proportional to the displacement $x$ of the movable fingers.

For small beam deflection (angle $<5^{\circ}$ ), we can consider the accelerometer as simplified spring-mass model. Assume the total sensing mass of the accelerometer as $M_{s}$, the inertial force $F_{\text {inertial }}$ experience by the sensing mass for acceleration $a$ along sensitive direction is:

$F_{\text {inertial }}=-M_{s} \cdot a$

Take for the total spring constant of the beams as $K_{\text {total }}$, the displacement $\boldsymbol{x}$ of the movable mass can be calculated as:

$x=\frac{F_{\text {inertial }}}{K_{\text {total }}}=-\frac{M_{s} \cdot a}{K_{\text {total }}}$

The resonant frequency $f_{0}$ of the spring-mass system is given by (Xiong 2005):

$f_{0}=\frac{1}{2 \cdot \pi} \sqrt{K_{\text {total }} / M_{s}}$

The sensing mass $M_{s}$ of the accelerometer includes the seismic mass and all the movable fingers attached to it, can be expressed as (Sharma et al.):

$M_{s}=\rho \cdot h \cdot\left(W_{m} \cdot L_{m}+N_{f} \cdot W_{f} \cdot L_{f}\right)$

$\rho$ the density of poly-Si, $h$ device thickness.

Spring constant $K_{s}$ of one section of beam can be designed by:

$K_{s}=\frac{12 \cdot E \cdot I_{b}}{L_{b}^{3}}$ where $I_{b}$ is the inertial momentum of the beam.

The spring constant $K_{\text {fold }}$ of one folded beam (Gupta and Mukherjee 2012) is:

$K_{\text {fold }}=K_{s}=\frac{E \cdot h \cdot W_{b}^{3}}{2 \cdot L_{b}^{3}}$

Four folded beams are connected in parallel and have the same size. Thus, the total spring constant $K_{\text {total }}$ of the device is given by:

$K_{\text {total }}=4 \cdot K_{\text {fold }}=\frac{2 \cdot E \cdot h \cdot W_{b}^{3}}{L_{b}^{3}}$

\section{Sensitivity analysis}

From Eq. (21), the displacement of the device along the sensitive direction can be expressed as:

$x=\frac{M_{s} \cdot g}{K_{\text {total }}}=\frac{\rho \cdot g \cdot\left(W_{m} \cdot L_{m}+N_{f} \cdot W_{f} \cdot L_{f}\right) \cdot L_{b}^{3}}{2 \cdot E \cdot W_{b}^{3}}$

and the displacement sensitivity $\boldsymbol{S}_{\boldsymbol{d}}$ become:

$S_{d}=\frac{\rho \cdot\left(W_{m} \cdot L_{m}+N_{f} \cdot W_{f} \cdot L_{f}\right) \cdot L_{b}^{3}}{2 \cdot E \cdot W_{b}^{3}}$

Given a displacement of the movable mass and fingers where $\boldsymbol{x}$ is much smaller than the static capacitance gap $d_{0}$ the capacitance sensitivity $S_{c}$ can be expressed (Amini 2006; Xiong 2005) as:

$S_{C}=\frac{2 \cdot N_{f} \cdot \varepsilon_{0} \cdot h \cdot\left(L_{f}-\Delta L_{f}\right)}{d_{0}^{2}} \cdot S_{d}$

where $\Delta \boldsymbol{l}_{f}$ is the length not covered of mobile finger, and if it is considered that $\Delta l_{f}$ much lower $\left(\Delta l_{f} \approx 0\right)$ however: The capacitance sensitivity $S c$ is given (Selvakumar et al. 1996; Bais and Majlis 2008).

$$
\begin{aligned}
S_{C} & =\frac{2 \cdot \varepsilon_{0} \cdot N_{f} \cdot L_{f} \cdot h}{d_{0}^{2}} \cdot x \\
& =\frac{2 \cdot \varepsilon_{0} \cdot N_{f} \cdot L_{f} \cdot h}{d_{0}^{2}} \cdot \frac{M_{s} \cdot g}{K_{\text {total }}}
\end{aligned}
$$

\section{Presentations and analyzes studied model}

We use MATLAB software to calculate the displacement, capacitance and sensitivity.

For following data (see Table 1):

The structural thickness layer in this device is limited to be $6 \mu \mathrm{m}$. 
Table 1 Physical and geometrical parameters of the model

\begin{tabular}{ll}
\hline Parameters & Design \\
\hline Capacitance gap $d_{0}$ & $3 \mu \mathrm{m}$ \\
Device thickness $h$ & $6 \mu \mathrm{m}$ \\
Mass width $W_{m}$ & $80 \mu \mathrm{m}$ \\
Mass length $L_{m}$ & $200 \mu \mathrm{m}$ \\
Beam width $W_{b}$ & $3 \mu \mathrm{m}$ \\
Beam length $L_{b}$ & $270 \mu \mathrm{m}$ \\
Finger width $W_{f}$ & $3 \mu \mathrm{m}$ \\
Finger length $L_{f}$ & $160 \mu \mathrm{m}$ \\
Number of sensing fingers $N_{f}$ & 32 \\
Young's modulus of poly-Si & $1.72 \times 10^{11} \mathrm{~Pa}$ \\
The dielectric constant of air $\varepsilon_{0}$ & $8.854 \times 10^{-12} \mathrm{~F} / \mathrm{m}$ \\
The density of poly-Si $\rho$ & $2.33 \times 10^{3} \mathrm{~kg} / \mathrm{m}^{3}$ \\
Gravity acceleration g & $9.81 \mathrm{~m} / \mathrm{s}^{2}$ \\
Movable sensing mass $M_{s}$ & $0.43841 \mu \mathrm{g}$ \\
Spring constant $K_{\text {total }}$ & $2.8313 \mathrm{~N} / \mathrm{m}$ \\
\hline
\end{tabular}

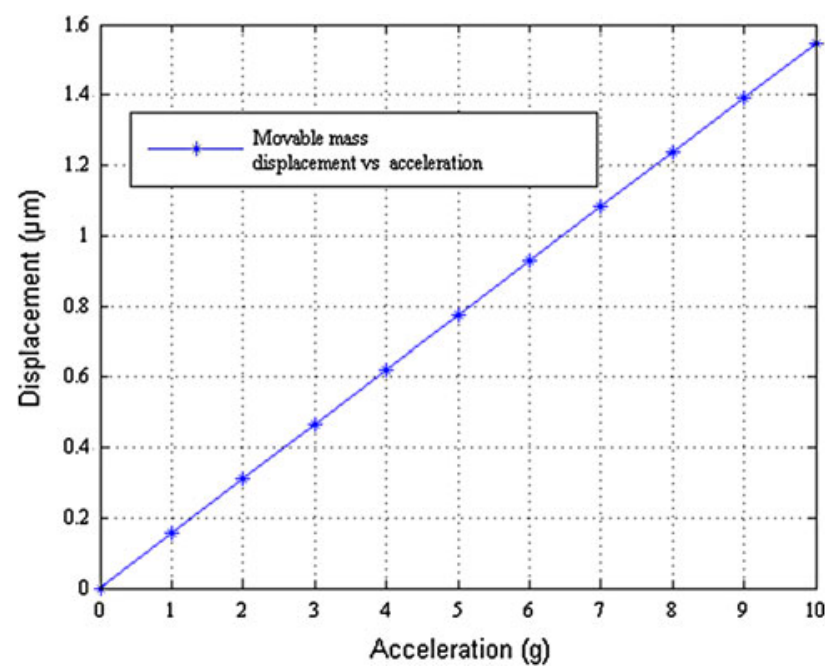

Fig. 9 Movable mass displacement vs acceleration

10.1 Movable mass displacement as a function of acceleration $x=f(g)$

The displacement's behaviour of movable mass as a function of acceleration with the basis of 0 up to $10 \mathrm{~g}$ by pad of $1 \mathrm{~g}$ is shown in Fig. 9 .

However, we can say that the increase in acceleration implies an increase in the displacement sensibility.

\subsection{Capacitance sensitivity $S_{c}=\mathrm{f}(\mathrm{x})$}

Knowing that $x$ depends on $g$ and $x \ll d_{0}$ then, the simulation of sensitivity as a function of movable mass displacement is given in Fig. 10: we note that the difference in capacity is extremely sensitive at the minimum displacement of the mobile fingers that explains the effectiveness of the model.

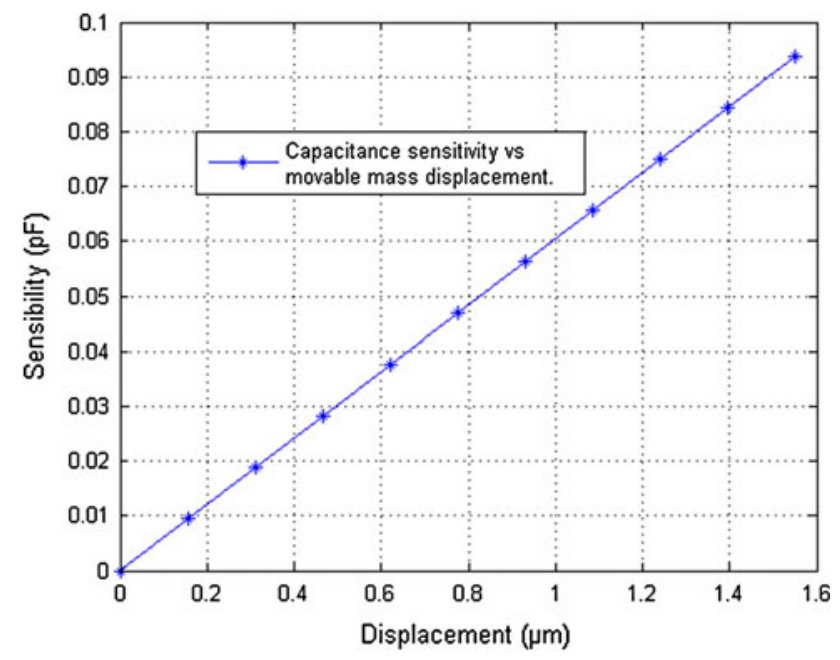

Fig. 10 Capacitance sensitivity vs movable mass displacement

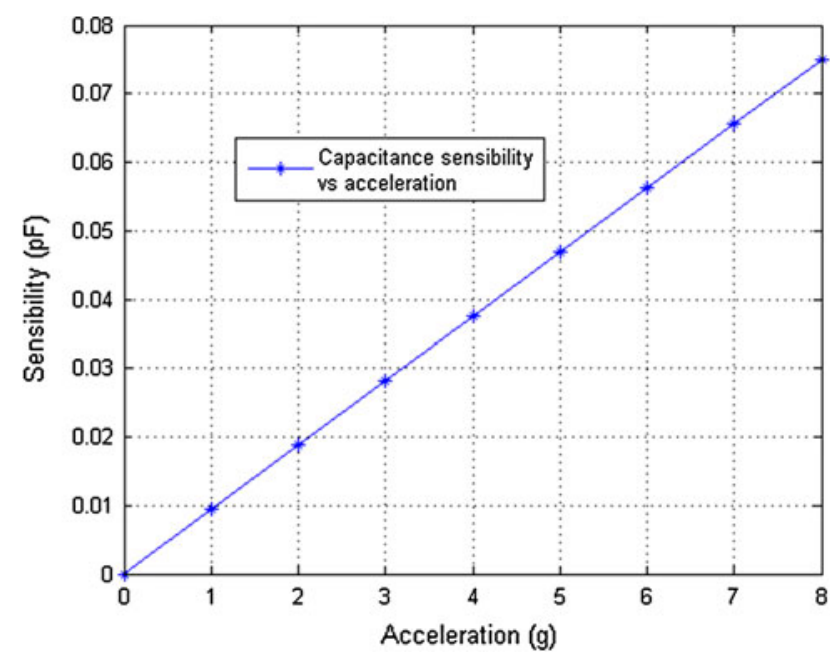

Fig. 11 Capacitance sensibility vs acceleration

10.3 Capacitance sensitivity $S_{c}=\mathrm{f}(\mathrm{g})$

A graphics presentation of capacitance sensitivity as a function of acceleration with the $0 \mathrm{~g}$ basis up to $8 \mathrm{~g}$ is shown in Fig. 11.

It is clear that the accelerations increase implies an increase in the capacitance sensitivity $S_{c}$; this proves that one can count on this model to obtain a high precision sensitivity.

10.4 A beam width effect on displacement

For different beam width $\mathrm{W}_{\mathrm{b}}(2,2.5,3 \mu \mathrm{m})$, it is noted that to have displacement $x$ inferior with gap $d_{0}$, it is necessary that acceleration $g$ does not exceed $5 \mathrm{~g}$ as shown in Fig. 12 . In this simulation $d_{0}$ is fixed at $3 \mu \mathrm{m}$. 


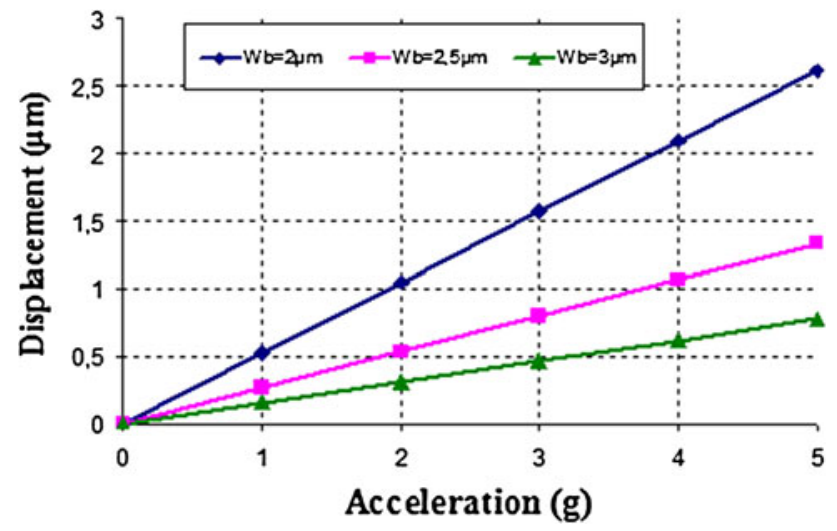

Fig. 12 Movable mass displacement vs acceleration with different $\mathrm{W}_{\mathrm{b}}$

In this case, the displacement of the folded beam comb accelerometer is inversely proportional to the third power of the beam width $W_{b}$.

\section{Conclusion}

After the results obtained by simulation of some parameters accelerometer capacitive, we note that the geometry of the component such as the width and the length of the mobile fingers as those of spring take a very important role on the acceleration sensitivity.

Theoretically, we can narrow down the beam width $W_{\boldsymbol{b}}$ to achieve very high device sensitivity. However, there is always a bottom limit for the beam width set by the minimum line width in a fabrication process. If the beam width is too narrow $<2 \mu \mathrm{m}$, it will become very challenging to fabricate the beam because the beam is extremely fragile and can be easily broken.

Therefore, to obtaining a good performance and a good sensitivity of a capacitive accelerometer, it is very important to choose better parameters such as the beam width and the beam length $\left(W_{b}\right.$ and $\left.L_{b}\right)$ which represents the suspension of the acceleration system.

Other share, the mobile fingers width and length $\left(W_{F}\right.$ and $L_{f}$ ) which constitute the capacities between the mobile fingers and the fixed fingers influence directly the value of these capacities then acceleration, which requires a choice very precise of these parameters.
Open Access This article is distributed under the terms of the Creative Commons Attribution License which permits any use, distribution, and reproduction in any medium, provided the original author(s) and the source are credited.

\section{References}

Amini BV (2006) A mixed-signal low-noise sigma-delta interface IC for integrated sub-micro-gravity capacitive SOI accelerometers. Dissertation, School of Electrical and Computer Engineering, Georgia Institute of Technology

Bais B, Majlis BY (2008) Low-g Area-changed MEMS accelerometer using bulk silicon technique. Institute of Microengineering and Nanoelectronics (IMEN), University Kebangsaan Malaysia 43600 UKM Bangi, Selangor, Malaysia. Am J Appl Sci 5(6): 626-632 ISSN 1546-9239

Gupta V, Mukherjee T (2012) Layout synthesis of CMOS MEMS accelerometers. Department of ECE, Carnegie Mellon University, Pittsburgh, PA-15213

Kuan LC (2008) Wireless MEMS accelerometer for real-time. Dissertation, Department of Electrical Engineering and Computer Science, Case Western Reserve University

Luo H, Zhang G, Carley LR, Fedder GK (2002) A post-CMOS micromachined lateral accelerometer. J Micro Electro Mech Sys 11(3):188-195

Selvakumar A, Ayazi F, Najafi K (1996) A high sensitivity Z-axis torsional silicon accelerometer. Center for Integrated Sensors and Circuits. University of Michigan, MI 48109-2122, The International Electron Devices Meeting (IEDM '96), San Francisco, CA

Sharma K, Macwan IG, Zhang L, Hmurcik L, Xiong X (2012) Design optimization of MEMS comb accelerometer. Department of electrical and computer engineering, University of Bridgeport, Bridgeport, CT 06604

Xie H, Fedder GK (2002) A CMOS Z-axis capacitive accelerometer with comb finger sensing. Department of Electrical and computer Engineering and the Robotic Institute, Carnegie Mellon University, Pittsburgh

Xiong X (2005) Built-in self-test and self-repair for capacitive MEMS devices. Department of Electrical and Computer Engineering and Computer Science of the College of Engineering, Division of Research and Advanced Studies of the University of Cincinnati, Dissertation

Zhang G (1994) Design and simulation of a CMOS-MEMS accelerometer. Carnegie Mellon University, Dissertation

Zhang G, Xie H, de Rosset LE, Fedder GK (1999) A lateral capacitive CMOS Accelerometer with structural curl compensation. Department of electrical and computer engineering and the Robotics Institute, Carnegie Mellon University, Pittsburgh, IEEE 1999 